EPJ Web of Conferences 59, 02008 (2013)

DOI: $10.1051 /$ epjconf/20135902008

(C) Owned by the authors, published by EDP Sciences, 2013

\title{
Design calculations for NIF convergent ablator experiments
}

\author{
R.E. Olson ${ }^{1}$, D.G. Hicks², N.B. Meezan², D.A. Callahan², O.L. Landen², \\ O.S. Jones ${ }^{2}$, S.H. Langer ${ }^{2}$, J.L. Kline ${ }^{3}$, D.C. Wilson ${ }^{3}$, H. Rinderknecht ${ }^{4}$, \\ A. Zylstra ${ }^{4}$ and R.D. Petrasso ${ }^{4}$ \\ ${ }^{1}$ Sandia National Laboratories, Albuquerque, New Mexico, USA \\ ${ }^{2}$ Lawrence Livermore National Laboratory, Livermore, California, USA \\ ${ }^{3}$ Los Alamos National Laboratory, Los Alamos, New Mexico, USA \\ ${ }^{4}$ Massachusetts Institute of Technology, Cambridge, Massachusetts, USA
}

\begin{abstract}
The NIF convergent ablation tuning effort is underway. In the early experiments, we have discovered that the design code simulations over-predict the capsule implosion velocity and shock flash $\rho \mathrm{r}$, but under-predict the hohlraum x-ray flux measurements. The apparent inconsistency between the $\mathrm{x}$-ray flux and radiography data implies that there are important unexplained aspects of the hohlraum and/or capsule behavior.
\end{abstract}

\section{INTRODUCTION}

In National Ignition Campaign (NIC) convergent ablation experiments, radiographs of an imploding capsule are analyzed to provide time-resolved measurements of shell radius, implosion velocity, mass remaining, and peak density [1,2]. An assessment of the accuracy of the NIC design code calculations is provided by comparing the simulation results to the experimental measurements. The integrated, two-dimensional, capsule-in-hohlraum simulations use the HYDRA [3] radiation-hydrodynamics code. Inputs to the simulations include measured target dimensions and densities; measured laser power; measured time-resolved SRS and SBS backscatter; adjustments to account for measured shock timing velocities; and calculated outer cone to inner cone laser cross-beam power transfer. The integrated HYDRA hohlraum-with-capsule simulations are combined with two-dimensional integrated LASNEX [4] calculations of the backlighter foil to provide simulated streaked radiographs that can be compared to the radiograph data. In addition, the HYDRA calculations are post-processed to provide simulations of the Dante x-ray flux measurements and the wedge range filter (WRF) proton spectrum measurements that are used to infer shock flash $\rho$ r [5].

\section{COMPARISONS OF EXPERIMENTAL AND SIMULATED X-RAY FLUX}

The recent NIC convergent ablation experiments employed two types of capsules - Ge-doped $\mathrm{CH}$ and $\mathrm{Si}$-doped $\mathrm{CH}$. The capsules had inner radii in the range of 909 to 935 microns, ablator thicknesses of 204 to 209 microns, a $6.3 \mathrm{mg} / \mathrm{cm}^{3}$ gas fill of 30\%D and $70 \% \mathrm{He}^{3}$, and were fielded in cryogenic "544 hohlraums" (5.44 mm diameter $\times 10.01 \mathrm{~mm}$ length) with Au walls and $0.96 \mathrm{mg} / \mathrm{cm}^{3} \mathrm{He}^{4}$ fill. The laser power history of the hohlraum drive was a $\sim 20 \mathrm{~ns}$ long variant of the standard NIF ignition pulse. Details involving the latter portion of the laser power are shown Fig. 1a. The plots labeled as "inner cone laser power", "outer cone laser power", and "total laser power" are experimentally-measured power histories.

This is an Open Access article distributed under the terms of the Creative Commons Attribution License 2.0, which permits unrestricted use, distribution, and reproduction in any medium, provided the original work is properly cited. 

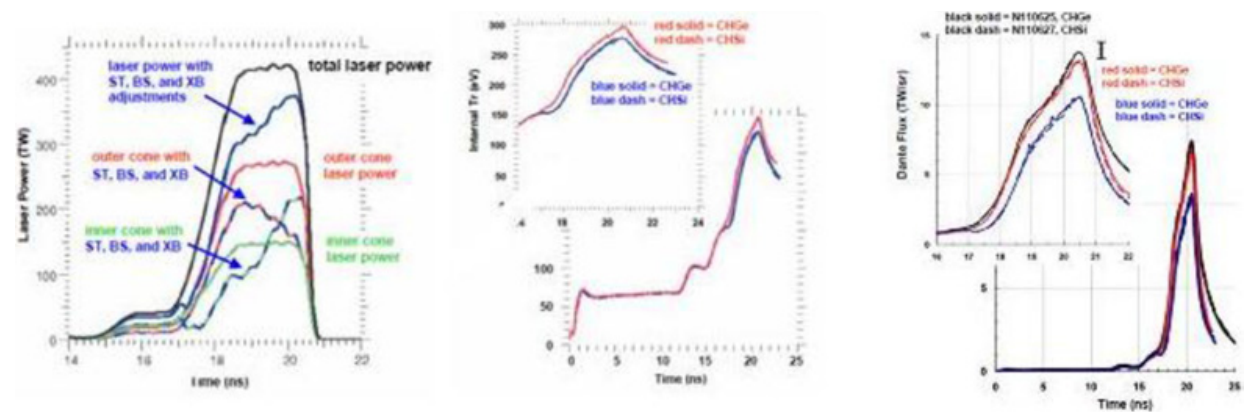

Figure 1. a) Laser power, b) internal radiation temperature, c) Dante x-ray flux.

These power histories are used as input for a preliminary integrated HYDRA simulation of the capsule and hohlraum. The plasma conditions from this integrated calculation are used as input for a cross-beam power transfer calculation, and the time-resolved inner and outer cone backscatter measurements are subtracted from the resulting power histories. The plots in Fig. 1a labeled "inner cone with ST, BS, and $\mathrm{XB}$ " and "outer cone with ST, BS, and XB" are the resulting power histories that also include small adjustments [6] in the early-time powers so as to match results of the shock timing measurements [7]. These laser power histories, appropriately divided into the 23 and 30 degree inner cones and the 44 and 50 degree outer cones, are used as inputs in the final integrated HYDRA simulation of the hohlraum with capsule.

Time-histories of the internal radiation temperature averaged over a region surrounding the outer edge of the capsule are indicated in Fig. 1b. As indicated in Fig. 1b, the solid plots are results of calculations using Ge-doped $\mathrm{CH}$ capsules, while the dashed plots use Si-doped CH capsules. As can be seen, the dopant type has very little effect on the internal radiation temperature history. The larger difference is between the red plots and the blue plots. The red plots are the results of "baseline" simulations using the laser input as shown in Fig. 1a. The blue, "modified" simulations incorporate an additional multiplier of 0.85 on the peak laser power (for times $>17 \mathrm{~ns}$ ). All of the simulations are postprocessed to obtain a simulation of the Dante x-ray flux measurement. Comparisons of the simulated and the measured $x$-ray flux histories are shown in Fig. 1c. In agreement with the simulations, the experimental measurements indicate that the dopant type has very little effect upon x-ray flux escaping the hohlraum (solid vs. dashed plots). The key differences are that the baseline simulations under-predict the flux measurements by about $6 \%$, while the modified ( $85 \%$ peak) simulations under-predict the Dante flux measurements by about $30 \%$.

\section{COMPARISONS OF EXPERIMENTAL AND SIMULATED RADIOGRAPHS}

Comparisons of lineouts from experimental and simulated radiographs are shown in Fig. 2. In Fig. 2a, the simulated radiograph from a baseline simulation of a Ge-doped $\mathrm{CH}$ capsule experiment is compared to the experimental radiograph data at three different times. It is clear that the capsule in the baseline simulation implodes too quickly. Fig. 2b shows a comparison of the simulated radiograph from a modified ( $85 \%$ peak) simulation with the experimental radiograph data. As can be seen, the modified simulation provides a much better match to the experimental radiograph. A similar comparison exercise for a Si-doped CH capsule experiment (for which the Dante data is also shown in Fig. 1c) indicates that a modified simulation using a $92 \%$ peak power multiplier provides a comparable match to the radiograph data.

The integrated simulations are also post-processed to provide time histories of the ablator remaining mass, the radius of the center of mass of the remaining mass, and the velocity of the center of mass of 


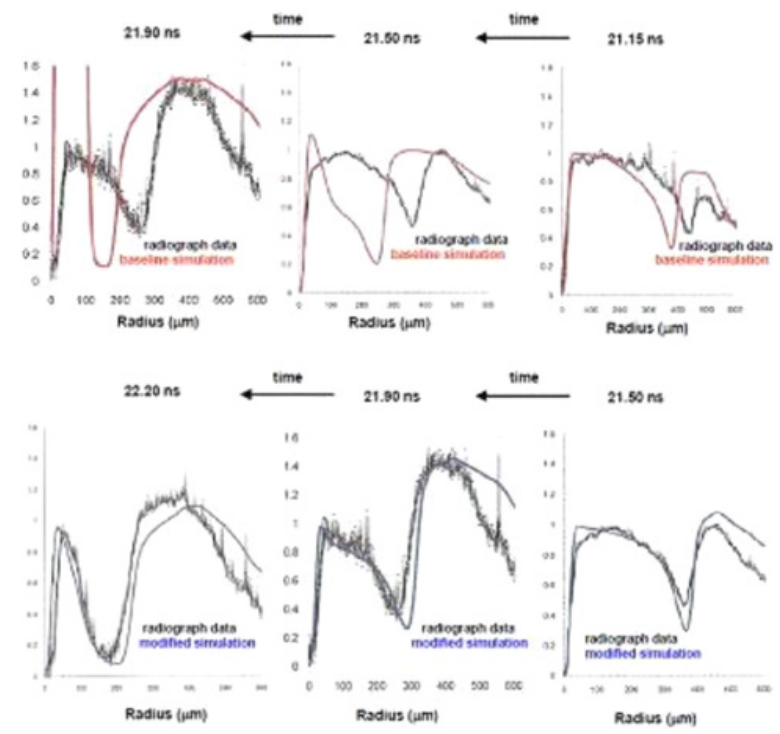

Figure 2. Comparison of lineouts from simulated radiographs and the radiograph data from a Ge-doped $\mathrm{CH}$ capsule experiment - a) baseline simulation; b) modified ( $85 \%$ peak) simulation.
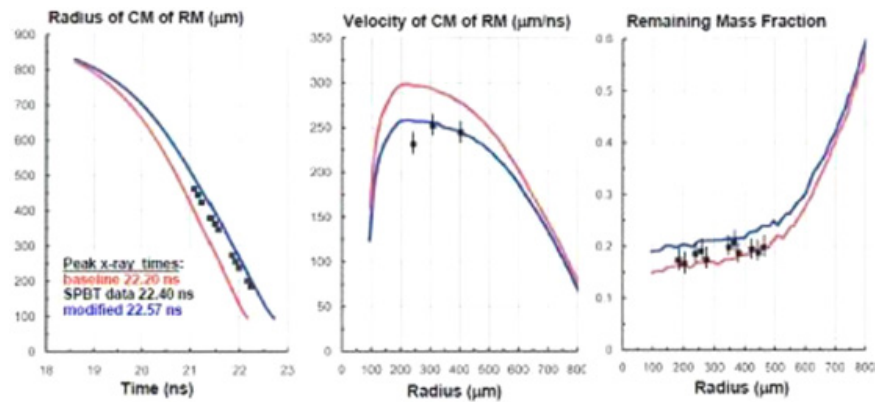

Figure 3. Comparison of baseline (red) and $85 \%$ modified (blue) simulations with parameters inferred via an xstreak analysis of the radiograph data from a Ge-doped $\mathrm{CH}$ capsule experiment.

the remaining mass. These plots are shown in Fig. 3 for the baseline and the modified calculations of the $\mathrm{Ge}$-doped $\mathrm{CH}$ experiment. Also shown in these plots are the values inferred via an "xstreak" analysis [8] of the radiograph data. As in the Fig. 2 comparisons, the xstreak analysis indicates that the capsule in the baseline simulation implodes too quickly, while the modified simulation ( $85 \%$ peak) is a better match to the data. The comparison of remaining mass fraction (Fig. 3c) in the simulations with the xstreakinferred values is inconclusive as to which is the better match with the data. A similar comparison of simulated radiographs with parameters inferred via an xstreak analysis of radiographs from Si-doped $\mathrm{CH}$ capsule experiments indicates that modified simulations with a $92 \%$ multiplier on the peak laser power provide a better match to the capsule implosion than does the baseline simulation - the same conclusion that was drawn from direct comparison of simulated and experimental radiograph lineouts.

\section{COMPARISONS OF EXPERIMENTAL AND SIMULATED PROTON DATA}

The simulated proton spectra for both the baseline and modified simulations have peaks that are about $1 \mathrm{MeV}$ lower than the peaks observed in the WRF data [5] (Fig. 4a). Only data from a Ge-doped 

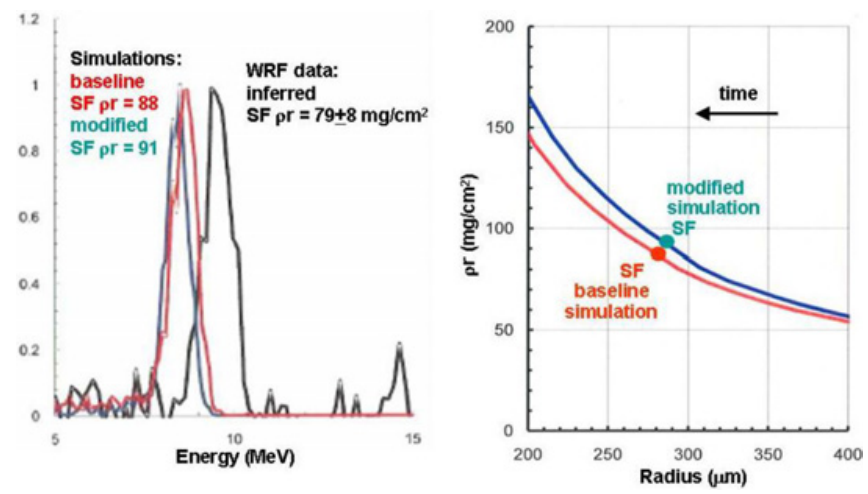

Figure 4. a) Simulated and measured proton spectra; b) simulated shock flash radius and $\rho$ r.

$\mathrm{CH}$ capsule experiment is shown in Fig. 4, but the proton spectra comparison is very similar in the Si-doped $\mathrm{CH}$ capsule experiments. The shock flash total $\rho$ r inferred from the measured proton spectrum is $79 \pm 8 \mathrm{mg} / \mathrm{cm}^{2}$. The baseline and modified simulations both tend to over-predict the shock flash $\rho$ r. As the shell converges, the total $\rho$ r increases. This is shown in Fig. $4 \mathrm{~b}$, along with points indicating the radius and $\rho$ r at which the shock flash occurs in the simulation. Eventually, the $\rho$ r inferred from the WRF will be combined with the radiography data and a shock flash time measurement [5] to further assess the accuracy of the simulation predictions.

\section{CONCLUSIONS}

Design code simulations of the NIF convergent ablation experiments tend to under-predict the x-ray flux measurements and over-predict the capsule implosion velocity. The apparent inconsistency between the $\mathrm{x}$-ray flux and radiography data implies that there are important unexplained aspects of the hohlraum and/or capsule behaviour. Possible explanations [9] might involve uncertainties in the Laser Entrance Hole (LEH) closure, LEH plasma, backscatter measurements, cross-beam power transfer, or opacities.

\section{References}

[1] D. G. Hicks et al., $7^{\text {th }}$ IFSA Conference, Bordeaux (2011)

[2] O. L. Landen et al., these proceedings

[3] M. M. Marinak et al., Phys. Plasmas 8, 2275 (2001)

[4] G. B. Zimmerman and W. L. Kruer, Comments Plasma Phys. Controlled Fusion 2, 51 (1975)

[5] R. D. Petrasso, et al., $7^{\text {th }}$ IFSA Conference, Bordeaux (2011)

[6] O. S. Jones, et al., these proceedings

[7] H. F. Robey, et al., these proceedings

[8] D. G. Hicks, et al., Phys. Plasmas 17, 102703 (2010)

[9] N. B. Meezan et al., these proceedings 